\title{
NON-ITERATIVE EXACT SIGNAL RECOVERY IN FREQUENCY DOMAIN OPTICAL COHERENCE TOMOGRAPHY
}

\author{
S Chandra Sekhar ${ }^{\dagger}$, Rainer A. Leitgeb*, Martin L. Villiger*, \\ Adrian H. Bachmann*, Thierry Blu ${ }^{\dagger}$, Michael Unser ${ }^{\dagger}$ \\ $\dagger$ Laboratoire d'Imagerie Biomédicale, ${ }^{*}$ Laboratoire d'Optique Biomédicale \\ Institute of Imaging and Applied Optics \\ École Polytechnique Fédérale de Lausanne, Lausanne, Switzerland \\ Emails: \{chandrasekhar.seelamantula, rainer.leitgeb, martin.villiger \\ adrian.bachmann, thierry.blu, michael.unser\}@epfl.ch
}

\begin{abstract}
We address the problem of exact signal recovery in frequency domain optical coherence tomography (FDOCT) systems. Our technique relies on the fact that, in a spectral interferometry setup, the intensity of the total signal reflected from the object is smaller than that of the reference arm. We develop a novel algorithm to compute the reflected signal amplitude from the interferometric measurements. Our technique is non-iterative, non-linear and it leads to an exact solution in the absence of noise. The reconstructed signal is free from artifacts such as the autocorrelation noise that is normally encountered in the conventional inverse Fourier transform techniques. We present results on synthesized data where we have a benchmark for comparing the performance of the technique. We also report results on experimental FDOCT measurements of the retina of the human eye.
\end{abstract}

\section{INTRODUCTION}

Optical Coherence tomography (OCT) is an effective and non-invasive interferometric technique that is well suited for the three-dimensional imaging of biological specimens. It has a penetration depth of 2-3 $\mathrm{mm}$ in tissue and offers micrometer resolution. One can distinguish between two primary types of OCT that differ in the way the optical signal is encoded. The earlier type is time domain optical coherence tomography (TDOCT) which uses a sequential scanning mechanism (mechanical displacement of a reference arm). The more recent type is frequency domain optical coherence tomography (FDOCT) that can acquire the information of the full depth scan within a single exposure [1]. FDOCT has a number of advantages that are making it increasingly popular. It is significantly faster than TDOCT and has a higher sensitivity [2]. It is also amenable to high-resolution imaging by using the principles of phase-shifting interferometry $[3,4]$. So far, the primary applications of FDOCT have been in dermatology and opthalmology [5]. The field is still quite new: the first medical images were obtained in 1995 [1] and the first in-vivo FDOCT measurements were reported in 2002 [6].

FDOCT involves inverting the spectrum domain measurements; this is typically achieved by inverse Fourier transformation [7]. However, Fourier inversion is known to introduce artifacts. The field is still nascent and not much work has gone into the signal processing

A provisional patent has been filed by the EPFL based on the research results reported in this paper. aspects of FDOCT. The most recent result in this direction is the use of minimum-phase functions to develop a Fienup-like iterative reconstruction algorithm [8].

In this paper, we propose a new non-iterative technique that enables us to perform exact signal recovery.

\section{SIGNAL ACQUISITION}

In Fig.1, we show the FDOCT experimental setup for data acquisition. The output of the broadband light source is optically split into two beams, each of which is directed towards one arm of a Michelson interferometer. A broadband reference mirror is placed at the end of one arm, called the reference arm. The light reflected from the broadband mirror serves as the reference signal in the interferometric signal measurement. The object which needs to be imaged is placed at the end of the other arm called the object arm. The light signals reflected from the object and the reference mirror are combined in a collinear fashion by the beam splitter and directed to a photodetector system or an optical spectrum analyzer. The light that is scattered back from each scatterer within the object volume contributes to the interfering signal over the measurement duration. The photodetection system performs a spectrum decomposition of the signal and records it as a function of the wavelength. Thus, in the FDOCT setup, the depth information of the object is encoded as a spectral fringe pattern. As a result of the encoding mechanism, FDOCT systems do not require scanning of the reference through the depth of the object. If we wish to acquire the volumetric description of the specimen, we need to perform lateral scanning.

\section{ARTIFACTS IN SPECTRUM INVERSION}

Refer to the setup shown in Fig.1. The signal from the object consists of many elementary waves emanating from different depths $z$ within the object. Let $a(z)$ denote the object scattering amplitude as a function of the depth $z$. The plane wave reflected from the broadband mirror in the reference arm is denoted as $a_{R}$. The spectrometer performs a spectrum decomposition of the signal as a function of the wavelength. The measurement is then mapped onto the wavenumber scale. The wavenumber $k=\frac{2 \pi}{\lambda}$ where $\lambda$ is the wavelength. The 


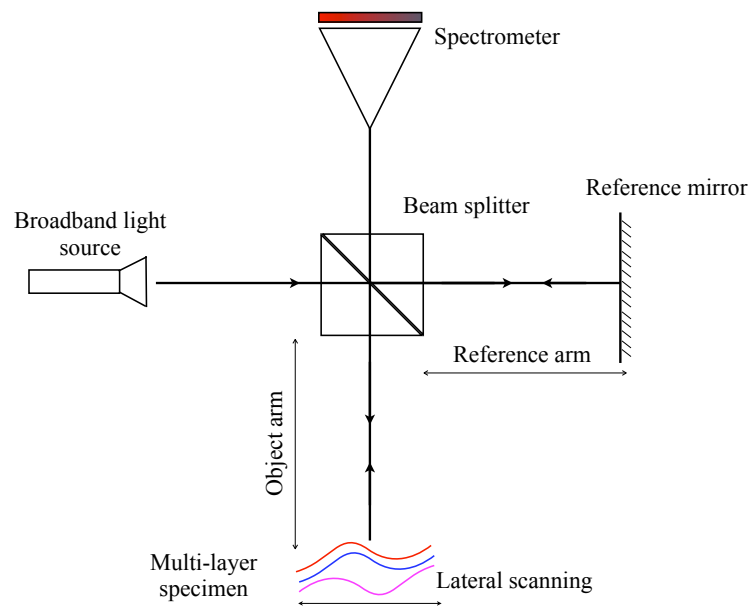

Fig. 1. Schematic of the Fourier domain optical coherence tomography system.

resulting intereference signal is denoted as $I(k)$ and is given by

$$
I(k)=S(k)\left|a_{R} e^{j 2 k r}+\int_{0}^{\infty} a(z) e^{j 2 k[r+n(z) z]} d z\right|^{2},
$$

where

$2 r$ is the pathlength in the reference arm,

$2(r+z)$ is the pathlength in the object arm,

$2 z$ is the pathlength in the object arm relative to the reference plane,

$n(z)$ is the refractive index as a function of the depth,

$a_{R}$ is the reference wave amplitude,

$a(z)$ is the amplitude of the wave backscattered from the object and

$S(k)$ is the spectrum intensity distribution of the broadband light source.

The zero-phase delay plane is assumed to be outside the sample. In the above equations, we have used the same notation as in [7] [Chapter 12 by Lindner et. al.]. We ignore the dispersion in the specimen. We also make a zeroth-order approximation: $n(z)=n$ since it is not straightforward to obtain the refractive index profile $n(z)$. This assumption is widely used and also holds when the bandwidth of the light source is moderate, which is often the case in practice. Without loss of generality, we set the reference wave amplitude to unity. Let us make the substitution $\omega=-2 k n$ to express the equations in the standard Fourier transform notation. We can now rewrite (1) as:

$$
I(\omega)=S(\omega)\left|1+\int_{0}^{\infty} a(z) e^{-j \omega z} d z\right|^{2}
$$

Thus, from the intensity measurements, we can only extract the pathlength differences which is exactly what we are interested in. Without loss of generality, we assume that $a(z)$ is real. We can expand the squares to obtain

$$
\begin{aligned}
I(\omega)= & S(\omega)\left(1+\int_{0}^{\infty} a(z) e^{-j \omega z} d z+\int_{0}^{\infty} a(z) e^{j \omega z} d z\right. \\
& \left.\int_{0}^{\infty} \int_{0}^{\infty} a(z) a\left(z^{\prime}\right) e^{-j \omega\left(z-z^{\prime}\right)} d z d z^{\prime}\right) .
\end{aligned}
$$

Apart from a constant offset, we have the following terms:

1. $\int_{0}^{\infty} a(z) e^{-j \omega z} d z$ known as the Müller fringe term,

2. $\int_{0}^{\infty} a(z) e^{j \omega z} d z$ which is the conjugate of the Müller fringe 3. $\int_{0}^{\infty} \int_{0}^{\infty} a(z) a\left(z^{\prime}\right) e^{-j \omega\left(z-z^{\prime}\right)} d z d z^{\prime}=\mathcal{F}\left\{r_{a a}(z)\right\}$ where $r_{a a}(z)$ is the autocorrelation of $a(z)$ describing the mutual interference of all elementary waves.

Since the source used is broadband, its point spread function is close to a Dirac. Otherwise, the point spread function of the source causes blurring of the above terms. The function $a(z)$ is one-sided and satisfies the property: $a(z)=0$ for $z<\left(z_{0}>0\right)$ where $z_{0}$ is the offset distance between the reference plane and the object surface. By taking the inverse Fourier transform of $I(\omega)$, we get a tomogram consisting of $a(z)$, its mirror image $a(-z)$, and $r_{a a}(z)$ which is the autocorrelation of $a(z) . a(z)$ and $a(-z)$ do not overlap with each other for a positive value of $z_{0}$. The autocorrelation $r_{a a}(z)$ is symmetric about $z=0$ and overlaps completely with $a(z)$ leading to artifacts and loss of resolution. If the object contains a highly dispersive medium, the overlap of $r_{a a}(z)$ and $a(z)$ can be very significant giving rise to several artifacts. These artifacts due to the overlap can be reduced by choosing a large value of $z_{0}$. However, a large value of $z_{0}$ causes the fringes in the power spectrum to get closer, requiring a higher resolution optical spectrum analyzer. For a given resolution of the spectrum analyzer, increasing $z_{0}$ limits the accessible depth in the object.

The problem of recovering $a(z)$ from $I(\omega)$, in an artifact-free manner has been addressed recently in [8]. The signal is assumed to be approximately minimum phase and iterative techniques are used for estimation. The type of problem has been addressed before in the digital signal processing literature [9]. If the desired signal is indeed minimum phase, we can recover it exactly from its magnitude spectrum. However, if it is not, we can recover only the minimumphase representation of the signal. Minimum-phase signal representations have had quite some success in the area of speech processing $[10,11,13]$. The inspiration for the present work comes from [11], but we go one step further and show that the practical interferometric signal acquisition model is such that it does not warrant any minimum-phase signal approximation and enables exact recovery.

\section{THE TECHNIQUE FOR EXACT SIGNAL RECOVERY}

Let us consider equation (2). The term $S(\omega)$ is the power spectrum of the source which can be measured separately by blocking the object arm. Hence, we can assume that it is known. Now, we can rewrite (2) as follows:

$$
\begin{aligned}
I(\omega)= & S(\omega)\left(1+\int_{0}^{\infty} a(z) e^{-j \omega z} d z\right) \\
& \left(1+\int_{0}^{\infty} a(z) e^{-j \omega z} d z\right)^{*} .
\end{aligned}
$$


We note that the autocorrelation term is a consequence of the multiplicative interaction of $\int_{0}^{\infty} a(z) e^{-j \omega z} d z$ with its conjugate. This can be transformed into an additive interaction, and to that extent a simpler one, by considering the logarithm of $I(\omega)$ :

$$
\begin{aligned}
\log I(\omega)= & \log S(\omega)+\log \left(1+\int_{0}^{\infty} a(z) e^{-j \omega z} d z\right) \\
& +\log \left(1+\int_{0}^{\infty} a(z) e^{j \omega z} d z\right) .
\end{aligned}
$$

We know that, in an interferometric signal acquisition setup, the intensity of the signal reflected from the object is much smaller than the reference intensity (which we assumed to be unity). Therefore, we can write $\left|\int_{0}^{\infty} a(z) e^{-j \omega z} d z\right|<1$. Henceforth, we denote $A(\omega)=\int_{0}^{\infty} a(z) e^{-j \omega z} d z$. Now, using the power series expansion: $\log (1+x)=\sum_{n=1}^{\infty}(-1)^{n-1} \frac{x^{n}}{n}$ for $|x|<1$, we can write:

$$
\begin{aligned}
\log I(\omega)-\log S(\omega) & =\sum_{n=1}^{\infty}(-1)^{n-1} \frac{A^{n}(\omega)}{n} \\
& +\sum_{n=1}^{\infty}(-1)^{n-1} \frac{A^{* n}(\omega)}{n}
\end{aligned}
$$

We note that $A(\omega)$ has a causal Fourier inverse and therefore $A^{*}(\omega)$ has an anti-causal Fourier inverse. Since $A(\omega)$ has a causal Fourier inverse, $A^{n}(\omega)$ has a causal Fourier inverse as well for $n \geq 1$. Similarly, $A^{* n}(\omega)$ has an anti-causal inverse for $n \geq 1$. Therefore, the inverse Fourier transform of $\log I(\omega)-\log S(\omega)$ is the sum of a causal and an anti-causal signal. Let us denote the inverse Fourier transform operation by $\mathcal{F}^{-1}$. The causal part of $\mathcal{F}^{-1}\{\log I(\omega)-\log S(\omega)\}$ is $\mathcal{F}^{-1}\{\log (1+A(\omega))\}$. In other words,

$$
\mathcal{F}^{-1}\{\log (1+A(\omega))\}=\mathcal{F}^{-1}\{\log I(\omega)-\log S(\omega)\} u(z),
$$

where $u(z)$ is the Heaviside/unit-step function. This allows us to write the following:

$$
A(\omega)=\exp \left(\mathcal{F}\left\{\mathcal{F}^{-1}\{\log I(\omega)-\log S(\omega)\} u(z)\right\}\right)-1 .
$$

Now, since $a(z)$ and $A(\omega)$ form a Fourier transform pair, we can compute one from the other. Thus, we have an explicit relation for $a(z)$ in terms of the measured intensities and hence this is an exact method for computing $a(z)$. We summarize the algorithm as follows:

1. Given the measurements $I(\omega)$ and $S(\omega)$, we compute the signal $c(z)=\mathcal{F}^{-1}\{\log I(\omega)-\log S(\omega)\} . c(z)$ is also known as the cepstrum [12] of $\mathcal{F}^{-1}\left\{\frac{I(\omega)}{S(\omega)}\right\}$.

2. Next, we retain only the causal part of the cepstrum $c(z)$. Let this be denoted as $c^{+}(z)$. Therefore, $c^{+}(z)=c(z) u(z)$.

3. We then compute the Fourier transform $C^{+}(\omega)$ of the causal cepstrum $c^{+}(z)$.

4. Next, we apply the transformation: $\exp \left(C^{+}(\omega)\right)-1$, which yields $A(\omega)$.

5. Finally, we compute $a(z)$ as $a(z)=\frac{1}{2 \pi} \int_{-\infty}^{+\infty} A(\omega) e^{j \omega z} d \omega$.

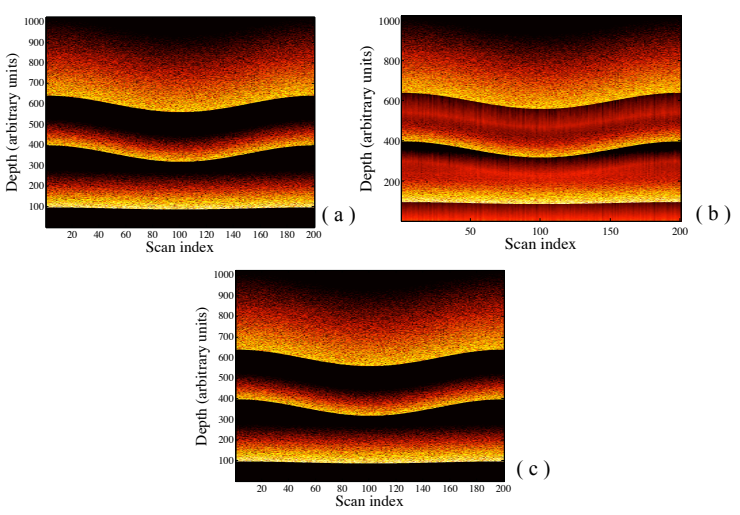

Fig. 2. (a) The synthesized scattering function of a three layer specimen, (b) the scattering function recovered by the inverse Fourier transform and (c) the scattering function obtained by using the new technique.

\section{EXPERIMENTAL RESULTS}

To validate the performance of our technique, we consider two sets of data: synthesized and experimental. We synthesized a multilayer biological specimen with the associated scattering function $a(z)$ as shown in Fig. 2(a). We have displayed two hundred lateral scans of the specimen. We assume that the source spectrum is perfectly flat. The signal recovered by the inverse Fourier transformation technique is shown in Fig. 2(b). Note that the artifacts due to the autocorrelation term corrupt the structural information of the specimen. The scattering function recovered by using the new technique is shown in Fig. 2(c). We note that the new technique yields exact and artifactfree signal recovery.

Next, we report the performance on experimental data. We describe the experimental setup in brief. We synthesized the broadband light source by using two superluminescent diodes (EXALOS) with center wavelengths $853 \mathrm{~nm}$ and $827 \mathrm{~nm}$. The respective full-widthat-half-maximum values (FWHM) are $34 \mathrm{~nm}$ and $25 \mathrm{~nm}$ resulting in an overall FWHM of $36 \mathrm{~nm}$, a central wavelength of $833.5 \mathrm{~nm}$ and an axial resolution of $8.5 \mu \mathrm{m}$ in air. The dispersion compensation in the reference arm matches sample arm optics as well as the water chamber of the eye. The light in the sample path passes an X-Y galvo scanning stage (Cambridge Technology) and illuminates the eye via a telescope with angular magnification of two. At the cornea, the optical power is $300 \mu \mathrm{W}$ and the beam waist is $1.8 \mathrm{~mm}$ resulting in a theoretical spot size of $14 \mu \mathrm{m}$ at the retina. The recombined reference and the sample arm light is guided through a single mode fiber to the spectrometer module. The latter is equipped with a volume transmission diffraction grating (Wasatch, 1200lines $/ \mathrm{mm}$ ) and an objective lens with focal length $135 \mathrm{~mm}$. The CCD line scan camera (ATMEL AVIIVA, 2048 pixel, 12bit) is driven at a line rate of $17.4 \mathrm{kHz}$ with an integration time of $\mathrm{t}=43 \mu \mathrm{s}$. The measured sensitivity is $98 \mathrm{~dB}$ close to the zero-offset distance.

To estimate the source spectrum, we averaged over all the scans. This yields a reasonably accurate estimate since the specimen-specific fringe pattern gets averaged across the incoherent lateral scans. We processed the data by both techniques. In the Fourier inversion technique, it is common practice to suppress the source term (background) by subtraction to obtain an image with higher contrast. We scaled the resulting images to possess the same dynamic range. The 

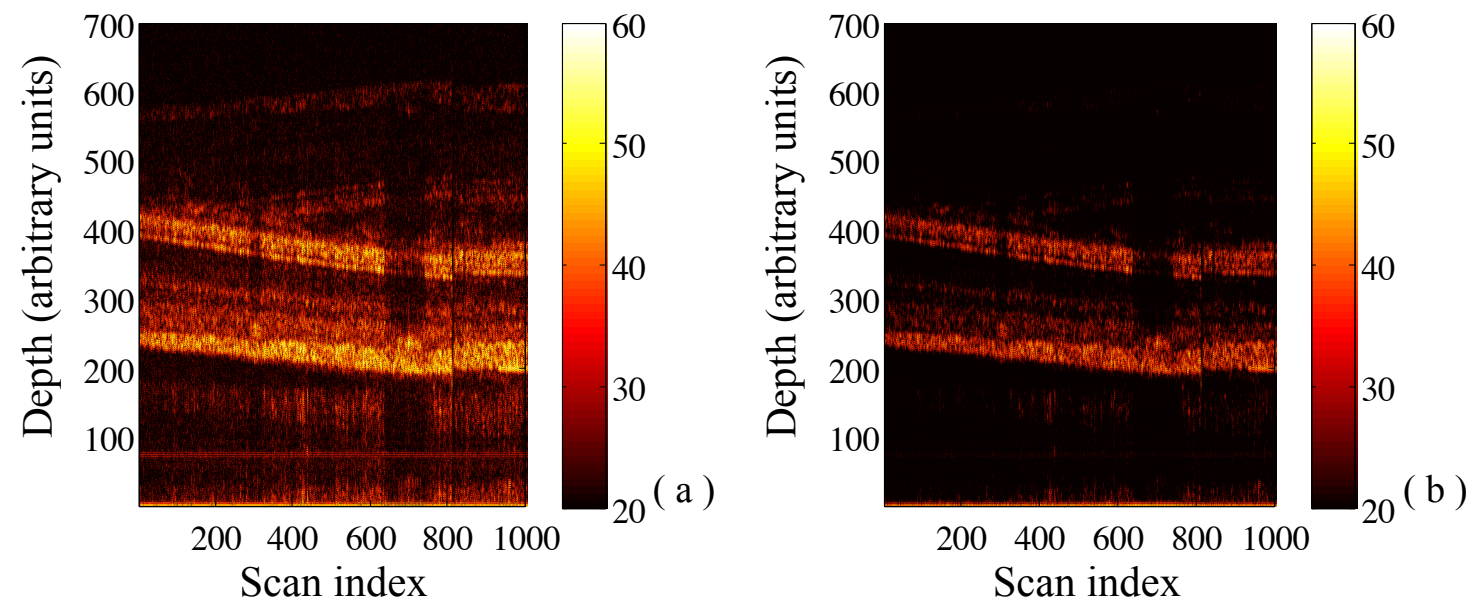

Fig. 3. (a) The tomogram obtained by the inverse Fourier transform and (b) the tomogram obtained by using the new technique.

resulting images are shown in Fig 3(a) and (b). Since the CCD camera noise floor was about $40 \mathrm{~dB}$ below the signal level, we show only the $40 \mathrm{~dB}$ dynamic range. We note a clear suppression of the autocorrelation artifacts. The structure of the specimen is also neatly resolved.

\section{CONCLUSION}

In this paper, we proposed a new technique for exact signal recovery in a FDOCT system. The new technique is non-iterative, non-linear and offers an exact solution unlike other approximate iterative/noniterative solutions. We demonstrated its performance on synthesized as well as experimental data and showed that it is superior to the inverse Fourier transformation technique. We also need to study the performance of the technique in the presence of additive noise for various noise levels. Our technique is also applicable to other interferometric measurements and generalizable to multidimensional signals.

\section{Acknowledgments}

This work was supported partly by the Center for Biomedical Imaging (CIBM) of the Geneva-Lausanne universities and the EPFL, and the foundations - Leenards and Louis-Jeantet.

\section{REFERENCES}

[1] A. F. Fercher, C. K. Hitzenberger, G. Kamp and S. Y. El-Zaiat, "Measurement of intraocular distances by backscattering spectral interferometry”, Opt. Comm., 117, pp. 43-48, 1995.

[2] R. Leitgeb, C. K. Hitzenberger and A. F. Fercher, "Performance of Fourier domain vs. time domain optical coherence tomography", Optics Express, Vol. 11, No. 8, pp. 889-894, 21 April 2003.

[3] R.A. Leitgeb, C.K. Hitzenberger, A.F. Fercher and T. Bajraszewski, "Phase-shifting algorithm to achieve high-speed long-depth-range probing by frequency domain optical coherence tomography”, Optics Letters, 28, 2201-2203, 2003.

[4] R. A. Leitgeb, C.K. Hitzenberger, A.F. Fercher and T. Bajraszewski, "Ultrahigh resolution Fourier domain optical coherence tomography", Optics Express, 12, 2156-2165, 2004.

[5] G. Hausler and M. W. Lindner, "Coherence radar and spectral radar - new tools for dermatological analysis", Jl. Biomed. Opt., 3, pp.21-31, 1998.

[6] M. Wojtkowski, R. Leitgeb, A. Kowalczyk, T. Bajraszewski and A. F. Fercher, "In-vivo human retinal imaging by Fourier domain optical coherence tomography", Jl. Biomed. Opt., 7, pp.457-463, 2002.

[7] B.E.Bouma and G.J. Tearney (Ed.), Handbook of optical coherence tomography, Marcel Dekker, Inc., 2002.

[8] A. Ozcan, M. J. F. Digonnet and G. S. Kino, "Minimum-phasefunction-based processing in frequency-domain optical coherence tomography systems", Vol. 23, No. 7, J. Opt. Soc. Am. (A), July 2006.

[9] T.F. Quatieri, Jr. and A.V. Oppenheim, "Iterative techniques for minimum phase signal reconstruction from phase or magnitude", IEEE Trans. Acoust., Speech and Signal Processing, Vol. 29, pp. 1187-1193, 1981.

[10] J. Makhoul, "Linear prediction: a tutorial review", Proc. IEEE, Vol. 63, No. 4, pp. 561-580, 1975.

[11] R. Kumaresan and A. Rao, "Model-based approach to envelope and positive instantaneous frequency estimation of signals with speech applications", Jl. Acoust. Soc. Am, 105(3), pp. 19121924, March 1999.

[12] D. G. Childers, D. P. Skinner and R. C. Kemerait, "The cepstrum: a guide to processing," Proc. IEEE, Vol. 65, No. 10, pp 1428-1443, Oct 1977.

[13] L.R. Rabiner and R.W. Schafer, "Digital processing of speech signals", Prentice Hall, 1978. 